\title{
1 Mechanisms of selection for the control of action in Drosophila melanogaster
}

3 Giovanni Frighetto ${ }^{1, *, \#}$, Mauro A. Zordan ${ }^{2,4, \#}$, Umberto Castiello ${ }^{1,4, \#}$ and Aram Megighian ${ }^{3,4, \#}$

4 'Department of General Psychology, University of Padova, 35131, via Venezia 8, Padova, 5 Italy.

$6 \quad{ }^{2}$ Department of Biology, University of Padova, 35121, via G. Colombo 3, Padova, Italy.

$7{ }^{3}$ Department of Biomedical Sciences, University of Padova, 35131, via U. Bassi 58/B,

8 Padova, Italy.

$9 \quad{ }^{4}$ Padova Neuroscience Center, University of Padova.

10 *Authors for correspondence (giovanni.frighetto@unipd.phd.it)

$11 \quad$ A All Authors partecipated equally to writing the manuscript

13 Running title: Mechanism of selection in Drosophila

14 Key words: Action selection, selective attention, motor re-orientation, model organism, 15 fixation, visuo-motor integration, Drosophila melanogaster

16 Summary statement: In this study we investigated adult fly locomotor behaviour in

17 response to distracting stimuli during free walking. Kinematic data reveal an interesting 18 phenomenon of motor re-orientation. 


\section{ABSTRACT}

In the last few years several studies have investigated the neural mechanisms underlying spatial orientation in Drosophila melanogaster. Convergent results suggest that this mechanism is associated with specific neural circuits located within the Central Complex (CC). Furthermore such circuits appear to be associated with visual attention, specifically with selective attention processes implicated in the control of action. Our aim was to understand how wild-type flies react to the abrupt appearance of a visual distractor during an ongoing locomotor action. Thus, we adapted the well-known 'Buridan paradigm', used to study walking behaviour in flies, so we could specifically address the mechanisms involved in action selection. We found that flies tended to react in one of two ways when confronted with a visual distractor during ongoing locomotion. Flies either: (i) committed to a new path situated midway between the original target and the distractor, consistent with a novelty effect; or (ii) remained on the original trajectory with a slight deviation in direction of the distractor. We believe that these results provide the first indication of how flies react, from the motor point of view, in a bi-stable context requiring the presence of selection-foraction mechanisms. Some considerations on the neural circuits underlying such behavioural responses are advanced.

\section{INTRODUCTION}

Living organisms have evolved neural information processing systems to allow interaction with the environment so as to maximize the probability of survival and reproduction. To reach this goal, appropriate information about the environment has to be extracted by perceptual systems in a form that can be used to guide actions (Tipper et al., 1998; Castiello, 1999). Visual attention systems appear to operate by mapping out relevant perceptual aspects of the environment and translating them into appropriate action control parameters. Drosophila melanogaster also seems to employ such mechanisms, for instance, in order to avoid predator attacks, to prevent collisions with obstacles or to head efficiently towards salient visual stimuli (Card and Dickinson, 2008; van Breugel and Dickinson, 2012; Maimon et al., 2008). Therefore, it is reasonable to assume that the presence of efficient action selection mechanisms constitute an evolutionarily conserved characteristic (Strausfeld and Hirth, 2013; Grillner and Robertson, 2016). The putative neural substrate of an action selection system in flies is thought to be contained within a doughnut-shaped structure called the ellipsoid body (EB) (Fiore et al., 2015), which is part of a wider ensemble of modular neuropils involved in locomotor behaviour termed the central complex (CC), (Strauss and 
Heisenberg, 1993; Martin et al., 1999; Pfeiffer and Homberg, 2014). Recently, using a two-photon calcium imaging technique, it has been shown that a class of $\mathrm{CC}$ neurons termed E-PG neurons having their dendritic tree in the EB and their axonal branches in the Protocerebral Bridge (PB) and Gall brain regions - are involved in tracking the orientation of a visual landmark and, to a lesser extent also the direction of body motion (Seelig and Jayaraman, 2015). The neurons of this circuit are arranged in a toroidal pattern, functionally subdividable into wedges. Each wedge responds to a particular direction of navigation through a mechanism involving a ring attractor dynamic model which explains how information concerning visual landmarks is integrated with self-motion in order to allow navigation (Turner-Evans et al., 2017; Heinze, 2017). Furthermore, this circuit is thought to be the neural centre for visual attention since it is characterized by a discrete single 'bump' of activity following the presentation of multiple visual stimuli (de Bivort and van Swinderen, 2016). This is reminiscent of a sort of attentional focus (Castiello and Umiltà, 1990; Castiello and Umiltà, 1992) and suggests a unified neurophysiological phenomenon which could form the basis of selection for the programming of locomotion direction.

Despite the above mentioned neurophysiological findings underlying landmark selection, little is known regarding the heading control in free moving adult flies. Horn and Wehner (1975) showed that walking flies faced with two stripes presented concomitantly and separated by an angular distance of less than $60 \mathrm{deg}$, preferred to move along the direction determined by the bisector of the angle between the two objects (Horn and Wehner, 1975). Conversely, when angles greater than $75 \mathrm{deg}$ were considered, the flies showed a distribution of orientations with two maxima directed toward either of the two stripes. This behaviour has been described in terms of a superposition of two turning-tendency functions, which are phase shifted according to the angle subtended by the landmarks (Poggio and Reichardt, 1973; Horn and Wehner, 1975). In the light of recent findings, suggesting that the E-PG neurons operate according to ring attractor dynamics (Kim et al., 2017), it might be speculated that, in the case of Horn and Wehner's experiment (1975), the 'compass needle' of the ring attractor points in a direction which is midway between the two landmarks. According to this idea, it has been observed that on some occasions E-PG activity transitioned from one offset to another relative to the two landmarks, indicating that this ambiguity may lead the fly to adopt an intermediate orientation (Seelig and Jayaraman, 2015). Thus, the turning tendency underlying fixation behaviour and the ring attractor model of the CC could be two sides of the same coin (Bahl et al., 2013; Seelig and Jayaraman, 2013).

With this in mind, we tested how the abrupt presentation of a visual stimulus to flies which are already engaged in locomotion (walking) toward a pre-existing visual target, would determine the activation of selection-for-action mechanisms which are then deployed in movement 
kinematics. To this end, we capitalized on an innate fly behaviour (i.e. unconditioned) in which flies continue to freely run back and forth between two opposing stripes inside a circular open arena (i.e. 'Buridan's paradigm'; Götz, 1980; Bülthoff et al., 1982; Strauss and Heisenberg, 1993; Strauss and Pichler, 1998). In our modified 'Buridan paradigm' a second stripe (with respect to the fly's visual field) was presented while the fly was already moving towards the pre-existing stimulus. We hypothesize that the appearance of the extra stripe might determine three possible scenarios: i) if the presence of the second stripe does not alter the originally programmed direction of locomotion, then the fly's movement should proceed in the direction of the first stripe, with no apparent changes along the path of the locomotion trajectory; ii) if the presence of the second stripe has a distracting effect, and therefore needs to be inhibited in order for the fly to proceed in the originally planned direction, then some evidence of this inhibitory process might be detectable in the form of slight perturbations in the locomotion trajectory; iii) if the presence of the second stripe determines the insurgence of an alternative motor program, which has the power to override the original one, then a dramatic change in direction toward the novel stripe should be evident. Surprisingly, the appearance of the novel target seemed to produce a tendency in the flies to turn towards a point midway between the two targets, as already shown by Horn and Wehner (1975). However, a more in depth analysis of the trajectories led us, in fact, to the identification of two alternative specific locomotor behaviours, namely that flies either: (i) committed to a new path situated midway between the original target and the distractor, consistent with a novelty effect; or (ii) presented a slight deviation of the original trajectory in the direction of the distractor. This in turn allows for interesting considerations regarding the nature of the selection-for-action mechanism in Drosophila melanogaster. In particular, the first type of response implies the abortion of the 'old' motor program in favour of a new one, while the second type of response suggests the deployment of an inhibitory mechanism operationalized in the form of slight trajectory changes.

\section{MATERIALS AND METHODS}

\section{Animals}

The experiments were performed on adult wild-type fruit flies (Drosophila melanogaster; Oregon$\mathrm{R}$ strain). All flies were reared on standard cornmeal-sucrose-yeast medium at $22^{\circ} \mathrm{C}$ in a $12 \mathrm{~h}$ light/12 h dark cycle at $60 \%$ relative humidity. Fly crowding was also controlled (20-30 flies each vial) to avoid competition for food. Only individual 2-5 day-old male flies were used. Flies were kept in their food vials until the beginning of the experiment. Thus for the experiment flies were not 
117 starved nor were their wings clipped. All experiments were conducted between zeitgeber time 2 and 1184 at room temperature $22-23^{\circ} \mathrm{C}$.

\section{Experimental setup}

120 To test how flies respond to the sudden appearance of new visual stimulus (distractor) during free

121 walking toward a fixed visual stimulus (block) we employed a cylindrical led-emitting-diode (LED)

122 modular display (Reiser and Dickinson, 2008) positioned around the fly (Fig. 1), and consisting in

12348 (12 x 4) LED panels (each panel made by an 8 x 8 array of LEDs) (IO Rodeo Inc, Pasadena, CA,

124 USA). A custom-designed transparent arena made of 3D-printed resin (iMaterialise HQ, Leuven,

125 BE, EU) was placed within the cylindrical LED display. The cylindrical LED display and the

126 transparent arena were in turn mounted on solid stainless steel brackets which were fixed to an

127 aluminium breadboard (Thorlabs Inc, Newton, NJ, USA). The setup was thus positioned on an anti-

128 vibration table, protected by a Faraday cage and covered with heavy black fabric. The arena

129 (maximum height at the centre $=3.5 \mathrm{~mm}$; diameter $=109 \mathrm{~mm}$ ) was designed so as to i) confine flies

130 in $2 \mathrm{D}$ space, ii) not allow the flies to reach the edge of the arena and iii) to impede flight by means

131 of a glass 'ceiling' (Simon and Dickinson, 2010). The arena was backlit by an infrared (IR) LED

132 array source (LIU850A, Thorlabs Inc, Newton, NJ, USA) and the IR light was diffused using paper

133 diffuser films placed between the IR light source and the arena. A CCD camera (Chameleon 3,

134 FLIR System Inc, Wilsonville, OR, USA) with 1288 x 964 pixel resolution, fitted with a 2.8-8 mm

135 varifocal lens (Fujifilm, Tokyo, JP) and an $850 \mathrm{~nm}$ band pass filter (MidOpt Inc, Woodwork Lane

136 Palatine, IL, USA) was mounted $36 \mathrm{~cm}$ above the arena in order to record fly activity. Videos of

137 flies moving in the arena were recorded at 21 frames $\mathrm{s}^{-1}$, following selection of a $700 \times 700$ pixel

138 region of interest which included the entire arena. In order to allow the experimenter to visually

139 observe all events occurring within the arena (including whether visual patterns were being

140 correctly displayed) an HD webcam (C310, Logitech, Lausanne, $\mathrm{CH}, \mathrm{EU})$ was also mounted

141 alongside the infrared camera.

\section{Procedure}

143 Flies were individually loaded into the arena with a mouth aspirator and were left to adapt in

144 complete darkness for at least 5 min. Individuals were then subjected to a 'Buridan's paradigm', by

145 illuminating two opposed bright stripes of 4 x 16 LEDs (width $\mathrm{x}$ height) each one covering $15 \mathrm{deg}$

146 of the fly's visual field when observed from the centre of the chamber. The classical interpretation

147 of the phenomenon underlying this paradigm refers to the alternation between fixation and anti-

148 fixation of attractive landmarks represented by black stripes on a bright background (Bülthoff et al., 
1982). Apparently, bright stripes on a dark background show no difference in terms of fixation

150 (Horn and Wehner, 1975; Seelig and Jayaraman, 2013). Preliminary experiments in our

151 experimental setup showed a more robust response to the 'Buridan's paradigm' in individuals tested

152 with bright stripes on dark background, and, therefore, we decided to run our experiments with this

153 setup. In our experiments, individual fly locomotion in 'Buridan's paradigm', consisting in the fly

154 continuously running to and fro between two opposing bright targets, was initially recorded for

155200 s (see Movie 1 in supplementary materials). Flies which did not exhibit this behaviour (i.e.

156 remained still or roamed at random) were not further considered (Kain et al., 2012). This allowed to

157 distinguish between flies that adopted a behaviour termed 'quantum search action' (i.e. a fixation

158 and anti-fixation strategy) from those which did not manifest such behaviour. In other words, this

159 procedure was aimed at selecting the 'searcher' phenotype considered for the following part of the

160 experiment (Bülthoff et al., 1982). At the end of this selection phase, the behavioural task-proper

161 was initiated. While the fly was still performing the 'Buridan paradigm', a second visual target

162 (distractor) was presented the moment the fly crossed the virtual midline of the arena while moving

163 between the two opposing bright stripes (a modified detour paradigm; Neuser et al., 2008).

164 Therefore, our task consisted in a classical 'Buridan paradigm' performed under two alternative

165 conditions. A distraction condition in which a single distracting-signal (chosen randomly among

166 four alternative signals) was presented concomitantly with the 'Buridan paradigm' stimuli,

167 whenever the individual crossed a virtual central window (27 mm width x $3.6 \mathrm{~mm}$ depth; see Fig. 2)

168 along the chosen path. From this point on we shall refer to this condition as the 'distractor'

169 condition. Distractors consisted in bright stripes of the same dimensions as the Buridan stripes (i.e.

$17015 \mathrm{deg}$ of the fly's visual field when viewed from the center of the arena). The distractors appeared

171 randomly to the right or left of the fly at an angle of either 30 or $60 \mathrm{deg}$ with respect to an ideal line

172 connecting the opposing Buridan stripes. Each time a fly crossed the virtual central window, the

173 distractor appeared for a 3 s period. During this period the two opposing Buridan stripes were

174 always present. A 'block' condition, instead, consisted in the presentation of the Buridan stripes

175 without any distractor. The experiment ended when the 'block' and the 'distractor' conditions had

176 been presented seven times (with an average experiment lasting $30 \mathrm{~min}$ ).

\section{Software and management}

178 The cylindrical LED display was controlled using available MATLAB (MathWork Inc, Natick,

179 MA, USA) scripts (Reiser and Dickinson, 2008). The MATLAB Image Acquisition Toolbox was

180 used to implement the system for video recording. Furthermore, in order to detect the position of

181 the fly's head in a specific spatial location (i.e. inside the virtual central window within the circular 
182 arena) and activate the necessary visual patterns on the LED panels accordingly, we implemented a

183 system for real time tracking using the FAST (Features from Accelerated Segment Test) method

184 (Rosten and Drummond, 2006) provided by the MATLAB Computer Vision System Toolbox.

185 Online tracking analysis, video recording and control of the LED arena were integrated into a single

186 custom GUI (Graphical Unit Interface), thus providing us with a unified software environment to

187 manage all experimental variables. All the scheduled events involved in each experiment were

188 automatically controlled by means of a custom script.

\section{Off-line tracking}

190 To obtain a greater definition of the fly's 2D position and body orientation we decided to track the

191 fly's trial recordings off-line using the CTRAX open source software (Branson et al., 2009). Errors

192 occurring during tracking were fixed manually using appropriate available MATLAB scripts

193 (CTRAX, FixErrors Toolbox) (Branson et al., 2009). Finally, other available MATLAB scripts

194 (CTRAX, Behavioral Microarray Toolbox) were used to compute a suite of speed and acceleration

195 properties (Branson et al., 2009).

196 Data pre-processing

197 The files obtained following the off-line tracking analysis pipeline described above were

198 transformed into .txt files, and imported into the R software (R Development Core Team, 2017)

199 environment for data pre-processing and an initial exploratory analysis by means of custom scripts.

200 For the trajectory analysis, only data from tracks in which single flies were directed towards the

201 target were selected (i.e. all tracks in the opposite direction were removed). The minimum track

202 length considered for analysis was $9 \mathrm{~mm}$ (i.e. 50 pixels; spatial resolution was 5.5 pixels per $\mathrm{mm}$ ).

203 Using this data frame (see Table 1 and Table 2) we performed track-centering. This operation

204 proved necessary due to the fact that, in order to trigger the appearance of the distractor and to start

205 the video recording, the flies had to cross a virtual central window within the circular arena. Given

206 the dimensions of this virtual window, the tracks showed scattered starting-points along the $\mathrm{x}$-axis

207 (width of the window), depending upon the point at which the fly entered the virtual window.

208 Therefore, since we were interested in evaluating the deviation of the fly locomotion paths caused

209 by the different distractors and since the body orientations of the flies were uniform among

210 conditions (Fig. 3A), we centered the starting point of all tracks at $\mathrm{x}=0$. Due to the limited depth of

211 the triggering window the starting y values appeared to be more homogeneously distributed among

212 the experimental conditions (Fig. 3B). Nonetheless, for uniformity, tracks were also centered at $y=$ 2130. 
Table 1. Velocity and Distance with respect to the experimental condition Condition No. trajectories

$$
\text { Velocity }\left(\mathrm{mm} \mathrm{s}^{-1}\right) \quad \text { Distance }(\mathrm{mm})
$$

$$
(\mu \pm \sigma)
$$

\begin{tabular}{llll}
\hline block & 123 & $18.83 \pm 13.62$ & $46.64 \pm 13.32$ \\
$30^{\circ} \mathrm{R}$ & 137 & $18.43 \pm 13.60$ & $48.23 \pm 12.74$ \\
$30^{\circ} \mathrm{L}$ & 135 & $18.50 \pm 13.87$ & $48.80 \pm 13.09$ \\
$60^{\circ} \mathrm{R}$ & 127 & $18.19 \pm 13.68$ & $47.41 \pm 14.33$ \\
$60^{\circ} \mathrm{L}$ & 131 & $18.44 \pm 13.54$ & $49.88 \pm 14.39$
\end{tabular}

Table 2. Number of trials by fly

\begin{tabular}{llllll}
\hline Fly ID & $\begin{array}{l}\text { No. } \\
\text { trajectories }\end{array}$ & Fly ID & $\begin{array}{l}\text { No. } \\
\text { trajectories }\end{array}$ & Fly ID & $\begin{array}{l}\text { No. } \\
\text { trajectories }\end{array}$ \\
\hline Fly_1 & 32 & Fly_8 & 30 & Fly_15 & 32 \\
Fly_2 & 34 & Fly_9 & 34 & Fly_16 & 29 \\
Fly_3 & 27 & Fly_10 & 32 & Fly_17 & 30 \\
Fly_4 & 30 & Fly_11 & 33 & Fly_18 & 27 \\
Fly_5 & 31 & Fly_12 & 34 & Fly_19 & 32 \\
Fly_6 & 33 & Fly_13 & 30 & Fly_20 & 31 \\
Fly_7 & 34 & Fly_14 & 31 & Fly_21 & 27 \\
\hline
\end{tabular}

\section{Statistical approach}

218 In order to understand how the presence of distractors explained the orientation and the trajectories

219 taken by the flies we tested a series of Linear Mixed Effects (LME) models using the R package

220 Ime4 (Bates et al., 2014). We used LME because such models allow to adjust estimates for repeated

221 sampling (i.e. more than one observation arises from the same fly) and for imbalance in sampling

222 (i.e. some flies are sampled more than others). LME also allow to take into account the

223 experimental variation (i.e. variation among flies or among other groupings within the data) and to

224 avoid the harmful effects of averaging, since this tends to remove variation (McElreath, 2016).

225 Subsequently, the LMEs were compared in order to select the best model (i.e. the best fit to the

226 data). For model selection we used the Bayesian Information Criterion (BIC) also known as the

227 Schwarz information criterion or Schwarz's BIC (Schwarz, 1978), an index that measures the

228 efficiency of the model in terms of data forecasting. Since BIC tends to favour models with fewer 
229

230

231

232

233

234

235

236

237

238

239

240

241

242

243

244

245

246

247

248

249

250

251

252

253

254

255

256

257

258

259

260

parameters, we further conducted a Bayes Factor (BF) analysis with a method based on the multivariate generalizations of Cauchy priors (JZS method, see Liang et al., 2008) using the $\mathrm{R}$ package BayesFactor (Morey and Rouder, 2015). We used this parameterization because it allows $\mathrm{BF}$ to have excellent statistical properties independently of the phenomenon under study (a method also known as 'objective Bayesian', see Wagenmakers, 2007). The Bayes Factor expresses the ratio between the plausibility of observed data under M1 (our best model) and the plausibility of observed data under M0 (the null model). We compared different models, and the one with the highest Bayes Factor (greatest plausibility) was selected. With the BayesFactor package, which inherits the MCMC (Markov Chain Monte Carlo) sampling algorithm from the R package coda (Plummer et al., 2006), we were also able to compute the posterior distributions of parameters (with 10000 iterations). This approach to estimating parameters enabled us to take maximum advantage of LME modelling, which provided the direct probability of an effect (i.e. posterior probability) as well as the computation of the evidence for the results. Analysis of variance (ANOVA) and nonparametric Mann-Whitney-Wilcoxon tests were also used, under the null hypothesis that the sample distributions belonged to the same population.

\section{RESULTS}

\section{Orientation effects}

As a first step we investigated the body orientation adopted by the flies at the time the second visual stimulus (i.e. the distractor) was presented. Before proceeding with this analysis we ascertained whether the flies maintained comparable velocity amplitudes across all conditions (conditions refers to the presence or absence of one of the four possible distractors). This was done in order to avoid any bias due to variations in velocity determined by the experimental conditions. We found no evidence for differences in velocity amplitude across conditions (Fig. 4A, B). Next, a series of LME models were fitted to the fly trajectory data (first two seconds following the presentation of the distractor) in order to obtain the best-fit model explaining the spatial orientation of flies as a function of time. The best fitting model (the one having the lowest BIC) was the following:

$$
Y=\beta_{1} X_{1}+\beta_{2} X_{1} X_{2}+\beta_{3} X_{2}+\lambda_{i}+\epsilon
$$

$Y$ represents the predicted orientation, $\beta_{1}$ and $\beta_{3}$ are the intercepts of regressions represented by the condition and time variables, respectively, while $\beta_{2}$ is the slope that represents the interaction between conditions and time. Finally, $\lambda_{i}$ represents the random effect which results in variation of the regression intercepts among trials within flies, while $\epsilon$ represents the error component. At a first glance, the linear regressions relating to the fixed effects (i.e. the orientation of flies in relation to 
261 the experimental condition) show that flies tend to orient consistently towards the distractor (Fig.

262 4C) suggesting an influence of the distractor on the orientation of the flies. However, as the data in

263 Fig. 4C also suggest, flies did not tend to turn fully towards the distractor. This can be more clearly

264 appreciated by evaluating a summary-measure of the orientation predicted by the LME model, that

265 is, the sum of the two coefficients $\beta_{1}$ and $\beta_{2}$ which in the LME model both refer to the experimental

266 condition effects (i.e. condition and condition-time interaction, respectively). This provides a more

267 direct and global representation of the change in orientation of flies following the presentation of

268 the distractors - showing that the orientation of flies does not precisely match the expected

269 orientation based on the position of the distractor (Fig. 4D). Rather, the model predicts that the

270 orientation of flies, following distractor presentation, is intermediate between the orientation of the

271 original trajectory and that of the distractor-influenced trajectory. Fig. 4E represents the distribution

272 of the random effects. Given the significant length of each experimental session (i.e. approx. 30

$273 \mathrm{~min}$ ) we also evaluated the possibility that flies could show signs of fatigue across trials, which in

274 turn might affect the re-orientation behaviour. Inspection of the average velocity profiles with

275 respect to time for each trial does not suggest the onset of fatigue, which would presumably result

276 in a systematic decrease in velocity as a function of time (supplementary material Fig. S1).

\section{Spatial trajectories}

278 Considering the idea that distractors could act on flies through a novelty effect, as a measure of the

279 flies' commitment to move towards the stimuli we explored the displacement of flies along the x-

280 axis at mid-path (i.e. after the flies had travelled $24 \mathrm{~mm}$ following the presentation of the

281 distractor). We hypothesized that, given the premise, there might be a reduction in the shift of the

282 flies' trajectory towards the new target whenever the target presented was (randomly) preceded by

283 one of the same kind (i.e. on the same side and at the same angle, in which case it would not be

284 interpreted by the fly as a novel stimulus). Interestingly, a tendency consistent with this idea could

285 in fact be observed (Fig. 5A). As a corollary, distinct left and right shifts (depending on the type of

286 distractor presented) were evident at the end of the paths (Fig. 5B), meaning that flies not only re-

287 oriented toward the distractor but that in so doing, they also committed to a new path (for individual

288 tracks see supplementary material Fig. S2). In order to obtain a model of the flies' trajectories,

289 which would provide an objective and quantitative evaluation of the strength and the extent of the

290 tendency of flies to shift their trajectories towards the distractors, we tested seven LME models

291 (Table 3). To this end we considered only trajectories at least $45 \mathrm{~mm}$ long, (which corresponds to

292 the radius of the surface of the arena effectively explorable by flies), were considered. The best

293 LME (i.e. the one with the lowest BIC), LME_6, was a very parsimonious model consisting of only 
294

295

296

297

298

299

300

301

302

303

304

305

306

307

308

309

310

311

312

313

314

one $\beta_{1}$ interaction parameter (representing the interaction between distance (d) and 'distractor' condition as a fixed effect, d:condition, Fig. 5C) in addition to a stochastic variation in the intercept among trials within flies (fly:trial, Fig. 5D) as a random effect:

$$
Y=\beta_{1} X_{1} X_{2}+\lambda_{i}+\epsilon,
$$

In this case, $Y$ represents the displacement of flies along the $\mathrm{x}$-axis. This implies that the best model represents effects as changes in the slope of the fitted line (which represents the interaction), according to the 'distractor' condition (Table 4). An estimate of the Confidence Intervals (CI) of the interaction parameters (Table 5) shows that none of them overlap which, in the classic frequentist perspective, implies a statistically significant difference between the effects of different conditions. The predictor $\left(\beta_{1}\right)$ can be converted into an angular measure by means of a simple trigonometric conversion:

$$
\beta_{1}=\sin ^{-1}\left\{\beta_{1} \cdot\left(\frac{180}{\pi}\right)\right\}
$$

and in this way it is possible to highlight the direction of flies predicted by the model (Fig. 5E). As already seen in the case of the fly body orientations, albeit to a lesser extent, the trajectories of the flies also shifted coherently with the distractor position (i.e. the greater the angle of the distractor with respect to the original trajectory, the farther the flies' path shifted in the direction of the distractor). None of the trajectories' regression per condition seems to predict an angulation (with respect to the fly) superimposable to the real angle subtended for both the 'block' and the 'distractor' conditions. Flies ended between the two but closer to the original target, with a little difference between the 30 and the 60 deg conditions.

Table 3. BIC of LMEs

\begin{tabular}{ccc}
\hline Model & df & BIC \\
\hline LME_6 & 7 & 137673.5 \\
LME_5 & 8 & 137678.9 \\
LME_7 & 12 & 137716.4 \\
LME_4 & 13 & 137721.6 \\
LME_3 & 9 & 147580.7 \\
LME_1 & 12 & 164847.1 \\
LME_2 & 7 & 164885.5 \\
LME_0 & 8 & 168269.8 \\
\hline
\end{tabular}


Table 4. Coefficients of the LME_6

\begin{tabular}{cccc}
\hline Parameter & Estimate & Std. Error & t value \\
\hline $\mathrm{d}$ :condition block & 0.0312009 & 0.0044081 & 7.078053 \\
$\mathrm{~d}:$ condition $30^{\circ} \mathrm{R}$ & -0.1638399 & 0.0040951 & -40.008641 \\
$\mathrm{~d}$ :condition $30^{\circ} \mathrm{L}$ & 0.2085999 & 0.0042346 & 49.261415 \\
$\mathrm{~d}:$ condition $60^{\circ} \mathrm{R}$ & -0.2733438 & 0.0045307 & -60.331208 \\
$\mathrm{~d}$ :condition $60^{\circ} \mathrm{L}$ & 0.3052126 & 0.0042944 & 71.072942 \\
\hline
\end{tabular}

Table 5. Estimated C.I. of parameters

\begin{tabular}{crr}
\hline Parameter & $2.5 \%$ & $97.5 \%$ \\
\hline sd_(Intercept)|fly:trial $\left(\sigma_{1}\right)$ & 5.7658491 & 6.5809188 \\
$\sigma$ & 3.9055042 & 3.9762879 \\
d:condition block & 0.0225617 & 0.0398401 \\
d:condition $30^{\circ} \mathrm{R}$ & -0.1718659 & -0.1558140 \\
d:condition $30^{\circ} \mathrm{L}$ & 0.2003008 & 0.2168990 \\
d:condition $60^{\circ} \mathrm{R}$ & -0.2822242 & -0.2644633 \\
d:condition $60^{\circ} \mathrm{L}$ & 0.2967963 & 0.3136289
\end{tabular}

319 The BF analysis highlighted a less parsimonious model with respect to the one which was selected

320 using the frequentist approach:

$$
Y=\beta_{1} X_{1}+\beta_{2} X_{1} X_{2}+\beta_{3} X_{2}+\lambda_{i}+\epsilon
$$

321 This model, in addition to a $\beta_{2}$ interaction term (d:condition), also presented the $\beta_{1}$ and $\beta_{3}$

322 parameters, which individually represent the effects of experimental condition and distance,

323 respectively. In the case of this model, the distribution of parameters and the goodness of fit were

324 evaluated (i.e. the standard error of residuals and the R-squared were estimated), in order to assess

325 the goodness of the model (Table 6). In this case, a "confidence interval" was computed, based on

326 the Highest Posterior Interval (HPI), using the R package TeachingDemos (Snow, 2016) (Table 7).

327 In practice, all points in an HPI region have a higher posterior density than points outside the

328 region. For this reason HPI is also called Highest Density Interval (HDI). Notwithstanding the

329 slightly more complex model produced by the BF analysis, this model provided essentially the same

330 general explanation for the experimental data as the LME model. Also in this case, none of the 
331 parameters bound to the 'experimental condition' variable showed any overlap in the predicted

332 values in terms of HPI (Fig. 6A), suggesting that the distractors produced significant deviations of

333 the flies' trajectories both in terms of shift and slope. The $\beta_{3}$ parameter (i.e. distance) showed a

334 non-significant shift in the intercept of the regressions (Fig. 6B), while the $\lambda_{i}$ random effect due to

335 the variation between flies appeared minimal (Fig. 6C). This analysis confirmed that flies respond

336 to distractors by shifting their locomotor trajectories essentially in accordance with the location of

337 the distractor, albeit not proportionately. In fact, with distractors presented at $30 \mathrm{deg}$ flies tended to

338 adopt a heading of $10 \mathrm{deg}$, while with distractors presented at $60 \mathrm{deg}$ flies adopted a heading of 16

339 deg.

340

Table 6. Model goodness of fit

\begin{tabular}{cc}
\hline Residual-se & R-squared \\
\hline 7.199331 & 0.4120791 \\
\hline
\end{tabular}

Table 7. HPI of parameters

\begin{tabular}{crr}
\hline Parameter & $2.5 \%$ & $97.5 \%$ \\
\hline distance & 0.0073964 & 0.0209496 \\
condition block & -0.0433319 & 0.3353007 \\
condition $30^{\circ} \mathrm{R}$ & -4.2541136 & -3.8968358 \\
condition $30^{\circ} \mathrm{L}$ & 3.8712083 & 4.2320447 \\
condition $60^{\circ} \mathrm{R}$ & -6.9712379 & -6.5951677 \\
condition $60^{\circ} \mathrm{L}$ & 6.4814631 & 6.8356365 \\
$\mathrm{~d}:$ condition block & -0.0303948 & -0.0031744 \\
$\mathrm{~d}:$ condition $30^{\circ} \mathrm{R}$ & -0.1868404 & -0.1609655 \\
$\mathrm{~d}:$ condition $30^{\circ} \mathrm{L}$ & 0.1671820 & 0.1942019 \\
$\mathrm{~d}:$ condition $60^{\circ} \mathrm{R}$ & -0.2949580 & -0.2669707 \\
$\mathrm{~d}:$ condition $60^{\circ} \mathrm{L}$ & 0.2775440 & 0.3045376 \\
$\sigma^{2}$ & 50.9114193 & 52.7319502 \\
\hline
\end{tabular}

\section{Kinematics indices}

343 The 'partial attraction' effect determined by the appearance of distractors led us to hypothesise that

344 perhaps a high number of the trajectories used in the model construction and analysis were

345 trajectories of flies which remained on the original straight path (i.e. which essentially did not 
respond to the distractor), impacting on the leverage of the model. Indeed, the raw distribution of the position of flies within the arena shows peaks which are consistent with the position of the original target (supplementary material Fig. S3). In order to clarify this issue we considered separately two situations: (i) the trials in which the distractor determined only a slight shift in the fly's trajectory in that direction, with the fly essentially maintaining the direction towards the original target (type 1); (ii) the trials in which the presence of the distractor determined a dramatic change in trajectory, with the fly abandoning the original direction in favour of the one dictated by the distractor (type 2). Type 2 trajectories were selected by considering a shift of at least $9 \mathrm{~mm}$ from an ideal straight line - orthogonal to the original target - at the time the individual crossed the middle of the path. This arbitrary procedure did not affect the balancing of the trials per condition in favour of one of the two types, maintaining a similar numerosity in the 'block' condition (Fig. 7A). Following this, a new parameter (i.e. shift) was introduced in the LME model as a third component of the interaction between distance and condition, thus increasing the values of the predictors (Fig. 7B). This kind of manipulation allowed us to investigate possible changes in kinematics following the appearance of the distractor. During the first 21 frames (i.e. $1 \mathrm{~s}$ ), the flies executed a fast turn in response to the distractor (Fig. 7C). In particular, around $250 \mathrm{~ms}$ the type 1 flies began to perform a body saccade in the contrary direction, while type 2 flies continued to maintain an orientation which was coherent with the distractor position (Fig. 7D). These fast turns did not affect the final trajectories of the flies (Fig. 7E).

\section{DISCUSSION}

The primary aim of this research was to evaluate in what way the abrupt presentation of different distracting visual stimuli to fruit flies which are already engaged in locomotion (walking) towards a pre-existing visual target, would influence the original locomotion action. Our results indicate that, following the presentation of a distractor, flies oriented their bodies according to a vector positioned midway between the original target and the distractor. Following the initial body orientation, flies then engaged in locomotion by committing to a new trajectory, essentially in one of two ways: (i) the presence of the distractor produced a slight perturbation in the original trajectory, but the ensuing movement then tended to proceed in the direction of the original target; (ii) the presence of the distractor determined the insurgence of an alternative motor program, which had the power to override the original one, leading to a dramatic change in the direction of the flies' motion. 


\section{Buridan with light stripes}

377 First and foremost some considerations concerning the use of the type of stimuli used here are in

378 order. By using a tethered flight simulator, it has been demonstrated that flies are usually attracted

379 towards long vertical bright or dark stripes, as an ethological reflex which guides flies towards

380 elements resembling vegetative perches (Maimon et al., 2008). Here we describe for the first time

381 the free walking behaviour of flies consisting of recurrent orientation inversions (i.e. alternation

382 between fixation and anti-fixation) between two diametrically opposed vertical bright stripes on a

383 dark background. Pioneer studies had shown that recurrent inversion is maximized with vertical

384 black stripes on a bright background (Bülthoff et al., 1982) and had considered the opposite contrast

385 as a repellent configuration for flies (Heisenberg and Wolf, 1979). Notwithstanding these earlier

386 observations, we observed a strong fixation response toward bright stripes in freely walking flies

387 consistent with more recent studies using tethered flying flies (Reiser and Dickinson, 2008;

388 Maimon et al., 2008). We are tempted to exclude that the recurrent direction inversions shown by

389 the flies in our case was due to anti-fixation, because when presented with the distractor stripes flies

390 were attracted to and maintained the distractor in front of them (suggesting fixation). Although the

391 functional distinction between flying and walking paradigms, as well as differences in the

392 experimental protocols, such as wing clipping (McEwen, 1918; Gorostiza et al., 2016), might be at

393 the basis of these contrasting findings, it is difficult to draw a coherent explanatory picture, and the

394 exact reason for the discrepancies remains as yet unknown. Rather, it is possible that the intensity of

395 the light used may have played a role in determining the discrepancies concerned with anti-fixation

396 behaviour of the flies, since in the case of LED displays (such as those used in the present study)

397 the maximum luminance reachable is $72 \mathrm{~cd} \mathrm{~m}^{-2}\left(\mathrm{~cd} \mathrm{~m}^{-2}=\mathrm{lux}\right)$ (Reiser and Dickinson, 2008), while

398 in the setups used in previous studies the luminance ranged between 300 and $1910 \mathrm{~cd} \mathrm{~m}^{-2}$ (Götz and

399 Wenking, 1973; Bülthoff et al., 1982; Virsik and Reichardt, 1976), which is at least 4 times higher.

400 This suggests that long vertical bars with high light intensities lead to avoidance, while long vertical

401 bars of lower brightness (i.e. in the region of $721 \mathrm{x}$ ) would represent an attracting stimulus, possibly

402 because under these conditions the bar appears similar to the reflectance of natural vegetation posts.

403 This hypothesis seems to be corroborated by a report of Heisenberg and Wolf (1984), in which a

404 grey background makes bright stripes as attractive as black stripes on a white background, while

405 bright stripes on a black background produce anti-fixation behaviour (Heisenberg and Wolf, 1984). 


\section{Novelty effect}

407 One aspect of the present results suggests that in our experimental paradigm the appearance of the

408 visual distractor stimuli produced a novelty effect in the flies. In particular this was manifested by 409 the re-orienting behaviour of the flies immediately following the appearance of the distractors. A 410 similar effect has been reported for tethered flying flies which showed a preference for a previously 411 uncued side of the arena when faced with bilateral stimuli (Shiozaki and Kazama, 2017). In neural 412 terms, it has been suggested that the Drosophila EB ring neurons (R4), are involved in determining 413 the slow turning tendency (i.e. body re-orientation) associated with this kind of visual experience.

414 Silencing of those neurons abolishes the innate behaviour for preferential orientation toward novel 415 stimuli (i.e. previously uncued sides) (Shiozaki and Kazama, 2017).

416 In another study using calcium imaging, the authors found that visual responses in ring neurons are 417 suppressed when competing stimuli are present in the contralateral visual field (Sun et al., 2017). In 418 this respect, contralateral suppression is hypothesized to act as a mechanism for location-based 419 stimulus selection by reducing the responses of ipsilateral stimuli in the presence of a second 420 stimulus. Furthermore, this suppressive effect appears to dependent upon short-term stimulus 421 history, specifically, ring neurons baseline activity showed a rebound after contralateral suppression, a phenomenon which could be involved in modulating the flies' subsequent visual responses to both ipsilateral and contralateral stimuli (Sun et al., 2017). Such evidence could partially explain our results, at least in terms of the novelty represented by the distractor.

The EB ring neurons - which innervate four concentric rings within the $\mathrm{EB}$ - appear to be retinotopically modulated by visual patterns but not by locomotor states (Seelig and Jayaraman, 2013). These neurons are possibly upstream from the EB wedge neurons, and convey visual information to the integrator layer. In fact, some of these neurons (R4d and R3) have been implicated in visual working memory (Neuser et al., 2008) and others (R4 and R1) in spacelearning linked to visual patterns (Ofstad et al., 2011) without affecting locomotor activity. Our

431 findings add to this literature by showing that flies are attracted by a novel visual stimulus and that 432 the attraction is manifested not only through a re-orienting of the body, but also by the ensuing 433 commitment of the individual to a new locomotor path.

\section{Reactive turning tendency}

435 Our data are consistent with the 'reactive turning tendency' described by Horn and Wehner (1975), 436 who noted that flies preferred to orient toward a position midway between two vertical stripes 437 placed at an angular distance less than $60 \mathrm{deg}$ (Horn and Wehner, 1975). In our paradigm, the 
sudden appearance of the distractor added a 'turning tendency' of the body to the one already engaged by shifting the internal compass needle toward the distractor. Differently from what reported by Horn and Wehner (1975), we observed that the trajectories did not lie midway between the original stimulus and the distractor, but instead remained closer to the former, meaning that the original stimulus had acquired the status of a stronger landmark. In our opinion, this behaviour might be the indication of a well-established motor program which is relatively 'impermeable' to the possible perturbation determined by the appearance of the distractor. This is in line with the observation that the E-PG neurons show a persistent activity maintaining the compass needle information even when the animal is in total darkness (Seelig and Jayaraman, 2015). The activity of such neurons remains linked to the position of a single vertical stripe even in the presence of a second identical stripe. Furthermore, the activity of such neurons does not always shift instantaneously following the abrupt displacement of a single visual target (Seelig and Jayaraman, 2015). Therefore, it would seem that the accomplishment of a coherent motor program requires locking on to a target.

\section{Selection for action via inhibition}

We were interested in understanding how flies detected and reacted to an abrupt distraction during the execution of a motor program. According to our original hypothesis we expected the distractor stimuli to determine an inhibitory or attracting behaviour acting upon already programmed trajectories, similarly to the interference effect observed in human and non-human primates under analogous circumstances (Tipper et al., 1998; Sartori et al., 2014; Bulgheroni et al., 2017). In these studies, participants were instructed to initiate a reaching movement after two stimuli (a target and a distractor) were presented. When the investigators compared a condition in which the target was presented alone with that in which there was a distractor acting as an alternative potential target, they found that the reaching path was affected in the latter case with the arm trajectory deviating away from or nearer to the distractor. This was observed even with regard to distractor objects that were unlikely obstacles to the reaching action. As those objects are also included in the initial processing of the whole context in which the action will be carried out, the motor program appropriate to reaching them is also produced in parallel, thus producing trajectory changes (Tipper et al., 1992; Tipper et al., 1997; Bulgheroni et al., 2017). This effect has been explained in terms of selective attention mechanisms mediating the selection of objects for action, with a specific mechanism acting to inhibit competing internal representations of distractor objects (Tipper, 1985; Tipper et al., 1992; Meegan and Tipper, 1998). Put simply, the effects caused by the presence of nearby objects seem to reflect inhibitory mechanisms. When the target is identified, the reaching 
movement towards the non-target is inhibited. But because there is an overlap between the target and the non-target(s), the act of reaching towards the target is affected by this non-target inhibition. Another crucial aspect of this model is that the amount of inhibition might be determined by the levels of activation of perceptual inputs. That is, inhibition is reactive such that its level is determined by the relative salience of the distractor. Thus distractors causing greater levels of neural excitation receive greater levels of inhibitory feedback. In the present circumstances our flies exhibited two kinds of behaviour in response to the distractor. The majority of flies fully espoused the new path dictated by the distractor. The remaining flies, maintained the original path with only a slight deviation toward the distractor. In both cases the flies acknowledged the presence of the distractor by making a fast saccade movement toward it within the first $250 \mathrm{~ms}$ from the onset of locomotion. This early fast saccade response could rely on the optomotor system, via the horizontal system neurons (HS; Bahl et al., 2013; Kim et al., 2015; Fujiwara et al., 2016). Nonetheless, to explain the present results our preferred idea is that inhibitory processes in Drosophila melanogaster occur at the level of the neuroanatomical structures involved in heading behaviour (Seelig and Jayaraman, 2015). This implies the involvement of the CC and in particular of the EB, with specific reference to the role played by dopamine in releasing and inhibiting motor programs. Similarly to what occurs in the mammalian brain (Grillner and Robertson, 2016), the signal involved in starting and halting an action sequence could be based on phasic dopamine release onto the EB in a manner similar to what is observed in the case of the nigrostriatal circuit of mice (Jin and Costa, 2010). The quantitative modulation of dopamine, via different receptors and/or perhaps through different types of neurons (Green et al., 2017), could engage and disengage the action programs, by respectively strengthening or weakening the inhibitory process. A high level of phasic release might enhance the specificity of action selection processes and movement initiation, while tonic release might inhibit the modules for action. This double mechanism would facilitate the emergence of motor responses from a repertoire of possible actions in order to readily cope with the sensory inputs determined by environmental variations. Fiore and collaborators (2015) suggest that a phasic dopamine release would allow the system to change the strength of the connections between sensory inputs and the EB, thus affecting the probability that the related motor action would be selected again. Conversely, a tonic release would not alter the connections' strength but would make the global system more stable (i.e. maintenance of selection) or unstable (i.e. sensitive to changes) depending on the receptor type involved (Fiore et al., 2015). However, it remains unclear how the system would differently weigh opposing pathways in order to regulate action selection. In this respect, our paradigm might provide a novel theoretical and methodological territory within which to classify and distinguish different mechanisms concerned with action 

selection in flies. Further research, considering the manipulation of the neuroanatomical circuit discussed above, is needed in order to dissect the neural mechanism underlying the action selection.

\section{ACKNOWLEDGMENTS}

We thank Fabian Feiguin (Neurobiology Lab, International Centre for Genetic Engineering and Biotechnology) for the Oregon-R fly stock, Paola Cisotto and Fortunato Piron for technical assistance.

\section{COMPETING INTERESTS}

The authors declare that they have no conflicts of interest with respect to their authorship or the publication of this article.

\section{FUNDINGS}

This study was supported by University of Padova DOR funds to AM and MAZ and Progetto Strategico funds (N. 2010XPMFW4) to UC.

\section{REFERENCES}

Bahl, A., Ammer, G., Schilling, T. and Borst, A. (2013). Object tracking in motion-blind flies. Nat. Neurosci. 16, 730-738.

Bates, D., Mächler, M., Bolker, B. and Walker, S. (2014). Fitting Linear Mixed-Effects Models using lme4. ArXiv14065823 Stat.

Branson, K., Robie, A. A., Bender, J., Perona, P. and Dickinson, M. H. (2009). High-throughput ethomics in large groups of Drosophila. Nat. Methods 6, 451-457.

Bulgheroni, M., Camperio-Ciani, A., Straulino, E., Sartori, L., D'Amico, E. and Castiello, U. (2017). Selective reaching in macaques: evidence for action-centred attention. Anim. Cogn. 20, 359-366.

Bülthoff, H., Götz, K. G. and Herre, M. (1982). Recurrent inversion of visual orientation in the walking fly, Drosophila melanogaster. J. Comp. Physiol. 148, 471-481.

Card, G. and Dickinson, M. H. (2008). Visually mediated motor planning in the escape response of Drosophila. Curr. Biol. CB 18, 1300-1307.

Castiello, U. (1999). Mechanisms of selection for the control of hand action. Trends Cogn. Sci. 3, 
264-271.

534 Castiello, U. and Umiltà, C. (1990). Size of the attentional focus and efficiency of processing.

535 Acta Psychol. (Amst.) 73, 195-209.

536 Castiello, U. and Umiltà, C. (1992). Splitting focal attention. J. Exp. Psychol. Hum. Percept.

537 Perform. 18, 837-848.

538 de Bivort, B. L. and van Swinderen, B. (2016). Evidence for selective attention in the insect brain. 539 Curr. Opin. Insect Sci. 15, 9-15.

540 Fiore, V. G., Dolan, R. J., Strausfeld, N. J. and Hirth, F. (2015). Evolutionarily conserved 541 mechanisms for the selection and maintenance of behavioural activity. Philos. Trans. R. Soc. Lond. 542 B. Biol. Sci. 370,.

543 Fujiwara, T., Cruz, T. L., Bohnslav, J. P. and Chiappe, M. E. (2016). A faithful internal 544 representation of walking movements in the Drosophila visual system. Nat. Neurosci.

545 Gorostiza, E. A., Colomb, J. and Brembs, B. (2016). A decision underlies phototaxis in an insect. 546 Open Biol. 6,.

547 Götz, K. G. (1980). Visual Guidance in Drosophila. In Development and Neurobiology of 548 Drosophila, pp. 391-407. Springer, Boston, MA.

549 Götz, K. G. and Wenking, H. (1973). Visual control of locomotion in the walking fruitfly 550 Drosophila. J. Comp. Physiol. 85, 235-266.

551 Green, J., Adachi, A., Shah, K. K., Hirokawa, J. D., Magani, P. S. and Maimon, G. (2017). A

552 neural circuit architecture for angular integration in Drosophila. Nature advance online 553 publication,.

554 Grillner, S. and Robertson, B. (2016). The Basal Ganglia Over 500 Million Years. Curr. Biol. CB 555 26, R1088-R1100.

556 Heinze, S. (2017). Unraveling the neural basis of insect navigation. Curr. Opin. Insect Sci. 24, 5855767.

558 Heisenberg, M. and Wolf, R. (1979). On the fine structure of yaw torque in visual flight

559 orientation of Drosophila melanogaster. J. Comp. Physiol. 130, 113-130.

560 Heisenberg, M. and Wolf, R. (1984). Vision in Drosophila: Genetics of Microbehavior (Studies of 561 Brain Function). Berlin, Heidelberg: Springer.

562 Horn, E. and Wehner, R. (1975). The mechanism of visual pattern fixation in the walking fly, 
Drosophila melanogaster. J. Comp. Physiol. 101, 39-56.

564 Jin, X. and Costa, R. M. (2010). Start/stop signals emerge in nigrostriatal circuits during sequence 565 learning. Nature 466, 457-462.

566 Kain, J. S., Stokes, C. and de Bivort, B. L. (2012). Phototactic personality in fruit flies and its 567 suppression by serotonin and white. Proc. Natl. Acad. Sci. U. S. A. 109, 19834-19839.

568 Kim, A. J., Fitzgerald, J. K. and Maimon, G. (2015). Cellular evidence for efference copy in 569 Drosophila visuomotor processing. Nat. Neurosci. 18, 1247-1255.

570 Kim, S. S., Rouault, H., Druckmann, S. and Jayaraman, V. (2017). Ring attractor dynamics in 571 the Drosophila central brain. Science.

572 Liang, F., Paulo, R., Molina, G., Clyde, M. A. and Berger, J. O. (2008). Mixtures of g Priors for 573 Bayesian Variable Selection. J. Am. Stat. Assoc. 103, 410-423.

574 Maimon, G., Straw, A. D. and Dickinson, M. H. (2008). A simple vision-based algorithm for 575 decision making in flying Drosophila. Curr. Biol. CB 18, 464-470.

576 Martin, J. R., Raabe, T. and Heisenberg, M. (1999). Central complex substructures are required 577 for the maintenance of locomotor activity in Drosophila melanogaster. J. Comp. Physiol. [A] 185, $578 \quad 277-288$.

579 McElreath, R. (2016). Statistical Rethinking: A Bayesian Course with Examples in R and Stan. 580 Boca Raton, FL, USA: Chapman and Hall/CRC Press.

581 McEwen, R. S. (1918). The reactions to light and to gravity in Drosophila and its mutants. J. Exp. 582 Zool. 25, 49-106.

583 Meegan, D. V. and Tipper, S. P. (1998). Reaching into cluttered visual environments: spatial and 584 temporal influences of distracting objects. Q. J. Exp. Psychol. A 51, 225-249.

585 Morey, R. D. and Rouder, J. N. (2015). BayesFactor: Computation of Bayes Factors for Common 586 Designs. R package version 0.9.12-2. Available at: https://CRAN.R587 project.org/package=BayesFactor/.

588 Neuser, K., Triphan, T., Mronz, M., Poeck, B. and Strauss, R. (2008). Analysis of a spatial 589 orientation memory in Drosophila. Nature 453, 1244-1247.

590 Ofstad, T. A., Zuker, C. S. and Reiser, M. B. (2011). Visual place learning in Drosophila 591 melanogaster. Nature 474, 204-207.

592 Pfeiffer, K. and Homberg, U. (2014). Organization and functional roles of the central complex in 
the insect brain. Annu. Rev. Entomol. 59, 165-184.

594 Plummer, M., Best, N., Cowles, K. and Vines, K. (2006). CODA: Convergence Diagnosis and 595 Output Analysis for MCMC, R News 6, 7-11.

596 Poggio, T. and Reichardt, W. (1973). A theory of the pattern induced flight orientation of the fly 597 Musca domestica. Kybernetik 12, 185-203.

598 R Development Core Team (2017). R: A Language and Environment for Statistical Computing. R 599 Foundation for Statistical Computing, Vienna, Austria. Available at: https://www.R-project.org/.

600 Reiser, M. B. and Dickinson, M. H. (2008). A modular display system for insect behavioral 601 neuroscience. J. Neurosci. Methods 167, 127-139.

602 Rosten, E. and Drummond, T. (2006). Machine Learning for High-Speed Corner Detection. In 603 Computer Vision - ECCV 2006, pp. 430-443. Berlin, Heidelberg: Springer.

604 RStudio Team (2017). RStudio: Integrated Development for R. RStudio, Inc., Boston, MA, USA. 605 Available at: https://www.rstudio.com/home/.

606 Sartori, L., Camperio-Ciani, A., Bulgheroni, M. and Castiello, U. (2014). Monkey see, monkey 607 reach: action selection of reaching movements in the macaque monkey. Sci. Rep. 4, 4019.

608 Schwarz, G. (1978). Estimating the Dimension of a Model. Ann. Stat. 6, 461-464.

609 Seelig, J. D. and Jayaraman, V. (2013). Feature detection and orientation tuning in the Drosophila 610 central complex. Nature 503, 262-266.

611 Seelig, J. D. and Jayaraman, V. (2015). Neural dynamics for landmark orientation and angular 612 path integration. Nature 521, 186-191.

613 Shiozaki, H. M. and Kazama, H. (2017). Parallel encoding of recent visual experience and self614 motion during navigation in Drosophila. Nat. Neurosci.

615 Simon, J. C. and Dickinson, M. H. (2010). A new chamber for studying the behavior of 616 Drosophila. PloS One 5, e8793.

617 Snow, G. (2016). TeachingDemos: Demonstrations for Teaching and Learning. R package version 618 2.10. Available at: https://CRAN.R-project.org/package=TeachingDemos/.

619 Strausfeld, N. J. and Hirth, F. (2013). Deep homology of arthropod central complex and 620 vertebrate basal ganglia. Science 340, 157-161.

621 Strauss, R. and Heisenberg, M. (1993). A higher control center of locomotor behavior in the 622 Drosophila brain. J. Neurosci. Off. J. Soc. Neurosci. 13, 1852-1861. 
623 Strauss, R. and Pichler, J. (1998). Persistence of orientation toward a temporarily invisible

624 landmark in Drosophila melanogaster. J. Comp. Physiol. [A] 182, 411-423.

625 Sun, Y., Nern, A., Franconville, R., Dana, H., Schreiter, E. R., Looger, L. L., Svoboda, K.,

626 Kim, D. S., Hermundstad, A. M. and Jayaraman, V. (2017). Neural signatures of dynamic

627 stimulus selection in Drosophila. Nat. Neurosci.

628 Tipper, S. P. (1985). The negative priming effect: inhibitory priming by ignored objects. Q. J. Exp.

629 Psychol. A 37, 571-590.

630 Tipper, S. P., Lortie, C. and Baylis, G. C. (1992). Selective reaching: evidence for action-

631 centered attention. J. Exp. Psychol. Hum. Percept. Perform. 18, 891-905.

632 Tipper, S. P., Howard, L. A. and Jackson, S. R. (1997). Selective Reaching to Grasp: Evidence

633 for Distractor Interference Effects. Vis. Cogn. 4, 1-38.

634 Tipper, S. P., Howard, L. A. and Houghton, G. (1998). Action-based mechanisms of attention.

635 Philos. Trans. R. Soc. Lond. B. Biol. Sci. 353, 1385-1393.

636 Turner-Evans, D., Wegener, S., Rouault, H., Franconville, R., Wolff, T., Seelig, J. D.,

637 Druckmann, S. and Jayaraman, V. (2017). Angular velocity integration in a fly heading circuit.

638 eLife 6,.

639 van Breugel, F. and Dickinson, M. H. (2012). The visual control of landing and obstacle

640 avoidance in the fruit fly Drosophila melanogaster. J. Exp. Biol. 215, 1783-1798.

641 Virsik, R. P. and Reichardt, W. (1976). Detection and tracking of moving objects by the fly

642 Musca domestica. Biol. Cybern. 23, 83-98.

643 Wagenmakers, E.-J. (2007). A practical solution to the pervasive problems of p values. Psychon.

644 Bull. Rev. 14, 779-804.

645 


\section{Fig. 1}

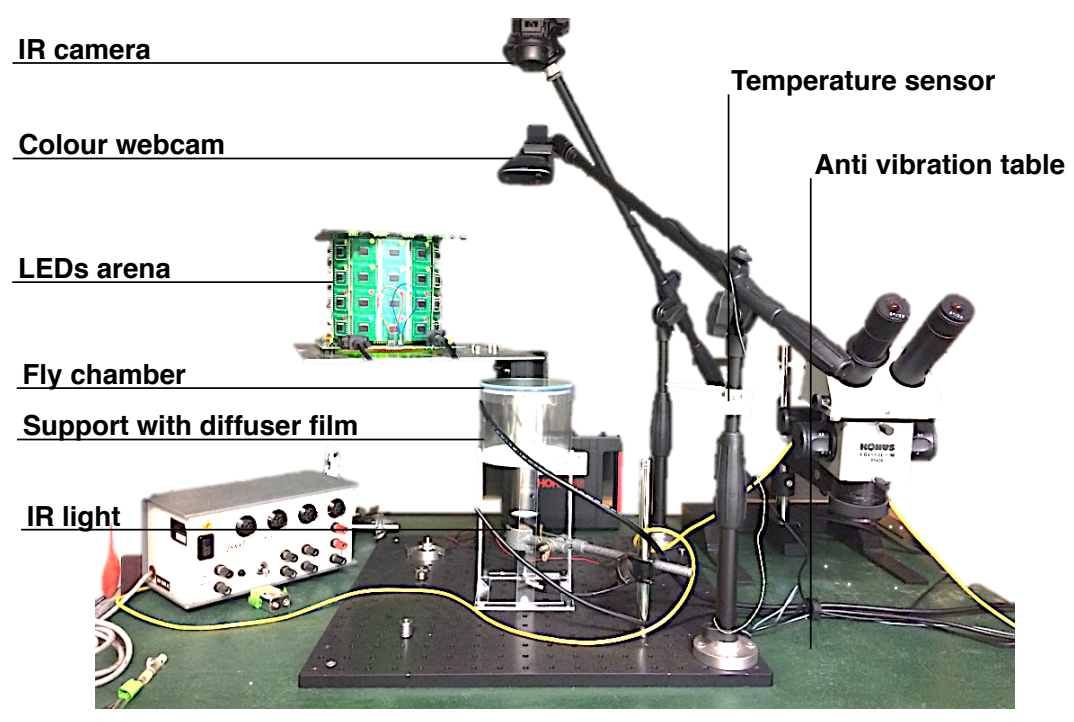

Fig. 1. Experimental setup. Image showing the main components of the setup utilized in the experiment described in the paper. 


\section{Fig. 2}
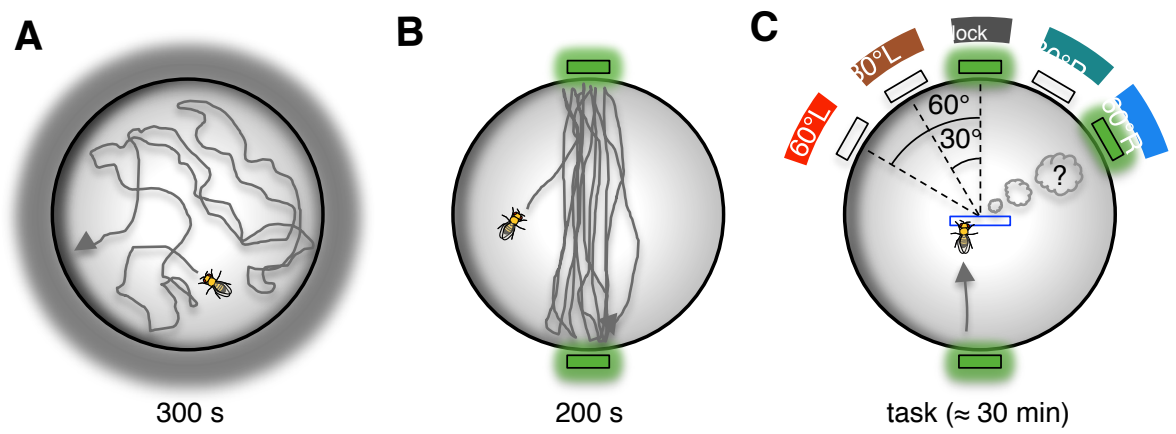

Fig. 2. Experimental procedure. Cartoon showing the three phases involved in each experiment. (A) Acclimatization period in complete darkness for $300 \mathrm{~s}$. (B) Two opposing bright green stripes were switched on and the behaviour was recorded for 200 s. (C) Behavioural task consisting in the random presentation of distracting visual stimuli (distractors) whenever the fly crossed a virtual central window (rectangle with blue borders). Behavioural task terminated when all five conditions were repeated seven times (about $30 \mathrm{~min}$ ). Distractors are represented as: 'block' for no distraction, ' $30^{\circ} \mathrm{R}$ ' and ' $60^{\circ} \mathrm{R}$ ' for distraction at 30 or $60 \mathrm{deg}$ on the right; ' $30^{\circ} \mathrm{L}$ ' and ' $60^{\circ} \mathrm{L}$ ' for distraction at $30 \mathrm{deg}$ and $60 \mathrm{deg}$ on the left. 


\section{Fig. 3}

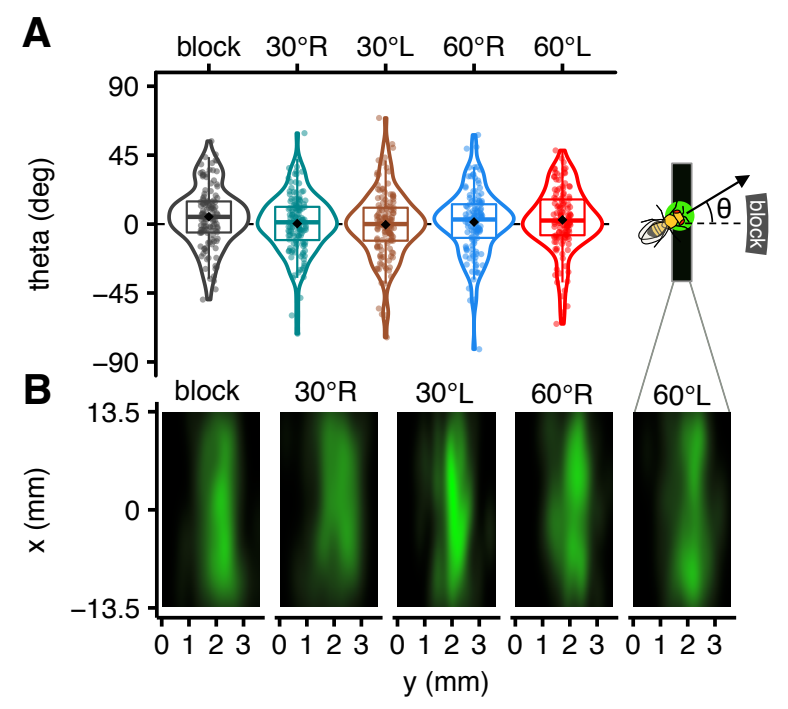

Fig. 3. Data inspection and trajectories centering. (A) Box-violin plot (i.e. box plot plus data distribution) of flies orientations (theta; see inset) in degrees at the exact moment of distractor presentation. Plots show that flies orientations when faced with the trials do not differ consistently among different conditions and are approximately normally distributed. Colour coding: black correspond to the absence of distractors (block); green to distractor at $30 \mathrm{deg}$ on the right side $\left(30^{\circ} \mathrm{R}\right)$; brown to distractor at 30 deg on the left $\left(30^{\circ} \mathrm{L}\right)$; blue to distractor at $60 \mathrm{deg}$ on the right $\left(60^{\circ} \mathrm{R}\right)$ and red to distractor at $60 \mathrm{deg}$ on the left $\left(60^{\circ} \mathrm{L}\right)$. Negative theta values refer to right-hand turns, while the positive ones to left-hand turns. The box-violin plot shows two measures of central tendency, the median in the box plot, and the mean of the data represented by the black square dot. (B) Heat map showing a density plot of all flies positions in the virtual rectangle when the distractor is presented. $\mathrm{x}$ and $\mathrm{y}$-axis are in $\mathrm{mm}$. 


\section{Fig. 4}

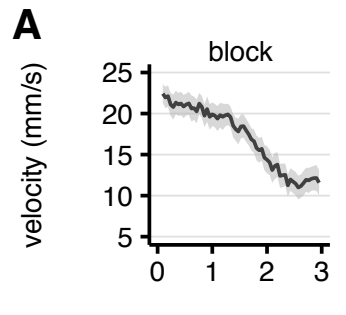

C

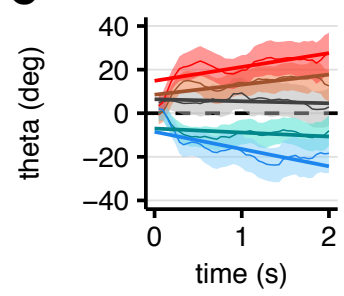

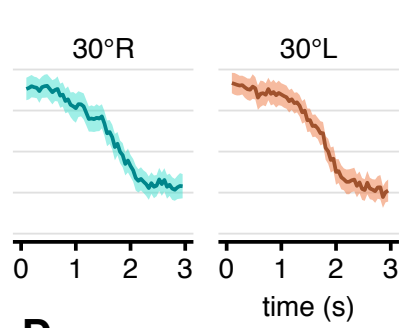

D

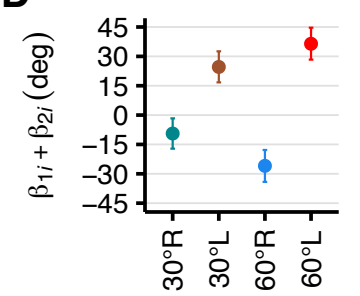

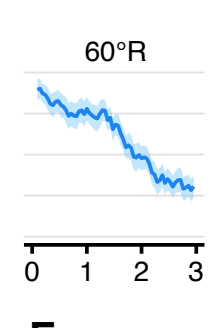

E

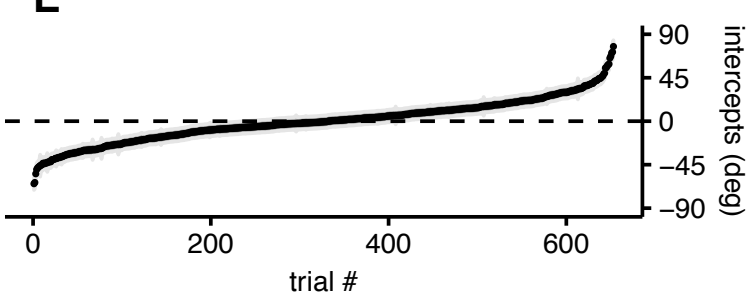

Fig. 4. Plots of orientations. (A) Velocity profiles from $t=0 \mathrm{~s}$ (crossing of the virtual central window; see Fig. 2C) to $t=3 \mathrm{~s}$ in the five conditions during the task. Shaded regions represent the s.e.m. (B) Box-violin plot (i.e. box plot plus the data distribution) of the velocity values in the five conditions. One-way ANOVA provides no evidenced of differences between mean velocities among the five conditions $\left(\mathrm{F}_{(4,31)}=.22, \mathrm{R}^{2}=.53, \mathrm{p}\right.$ $=.93$ ). (C) Plot of the mean orientation (theta) from $t=0$ to $t=2 \mathrm{~s}$ in the five conditions. Shaded regions represent s.e.m. Thick lines are regression lines for each condition. (D) Sum of the two coefficients $\beta 1$ and $\beta 2$ both referred to the condition effects (i.e. intercept and interaction with time), which allows to grasp the amount of change in orientation. (E) Random effect plot for each trial nested within flies (fly:trial). Dots represent the conditional means (also known as BLUPs, Best Linear Unbiased Predictions) while the shaded region (grey) corresponds to the standard deviations. In all images of the panel, the color-coding is as previously described (see Fig. 3). 
Fig. 5
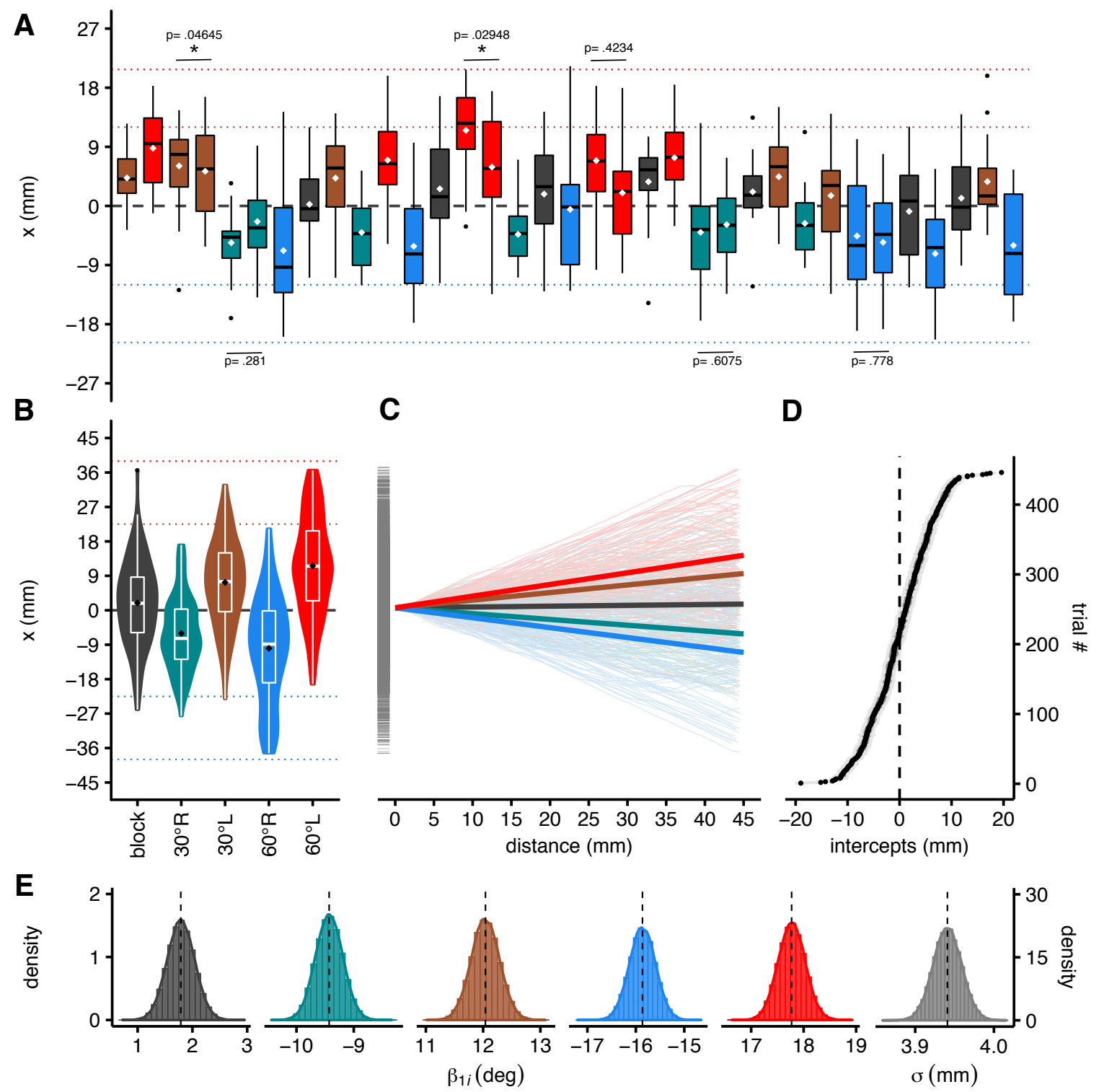


\section{Fig. 5}

Fig. 5. Plots of trajectories. (A) Box and whiskers plot of the displacement of flies along the $\mathrm{x}$-axis at mid-path (i.e. after the flies had travelled $24 \mathrm{~mm}$ following the presentation of the distractor). Each trial corresponds to a specific condition and is presented across time from left to right. This allows to appreciate the horizontal shift of the trajectories, at midway, between trials. This graph shows two measures of the central tendency, the median as a black horizontal line inside the box plot and the mean represented by a white squared dot. The vertical extension of each box represents the interquartile range (IQ). The whiskers extending from each box represent the extension of the data (i.e. max. and min. of the data within 1.5 times the IQ), while isolated black dots represent outliers. It can be observed that when the same distractor is immediately re-presented (which can only occur occasionally, due to the randomness of distractor presentation), the shift along the x-axis is usually smaller than the shift observed when the distractor is presented for the first time or has not been presented recently. Only on two occasions out of the six, did the mean displacement values between two successive presentations of the same distractor differ significantly $(p=.04645$ and $p=.02948)$. Statistical comparisons were done using the non-parametric Mann-Whitney-Wilcoxon test. (B) Box-violin plot (i.e. box plot plus the data distribution) of the displacement of flies along the $\mathrm{x}$-axis for each condition when flies have travelled for $45 \mathrm{~mm}$ (i.e. along the axis connecting the two fixed stimuli) by condition. (C) Plot of the regression lines (thicker lines) for each condition with the intercept fixed at $\mathrm{x}=0$ for all trajectories (thinner lines). (D) Plot of conditional modes of the random effects of the LME_6 model. Dots represent the conditional means (also known as BLUPs, Best Linear Unbiased Predictions) while the shaded region (grey) corresponds to the standard deviations. This represents the difference between the average predicted response for a given condition and the response predicted for a particular individual. (E) Approximate density profile of the probability density function for the sampling distribution for each parameter. The six distributions show the likelihoods of the five interaction parameters (between distance and condition), with $\sigma$ representing the residual standard deviation. In all images of the panel the color-coding is as previously described (see Fig. 3). 


\section{Fig. 6}

A

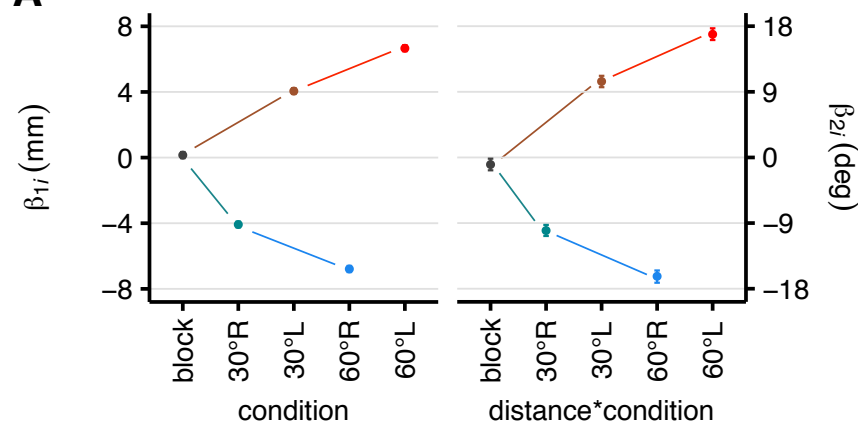

B

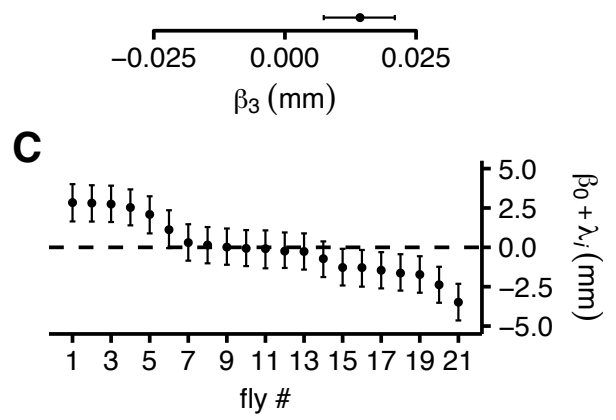

Fig. 6. HPI plot of parameters. (A) Fixed effects of condition parameter (on the left) and interaction parameter (on the right) between distance and condition with their $97.5 \%$ Highest Posterior Intervals (HPI). (B) Fixed effects of distance parameters with their 97.5\% HPI. (C) Random effects plot of the model represented for each fly. Colours encode conditions as previously described (see Fig. 3). 
Fig. 7

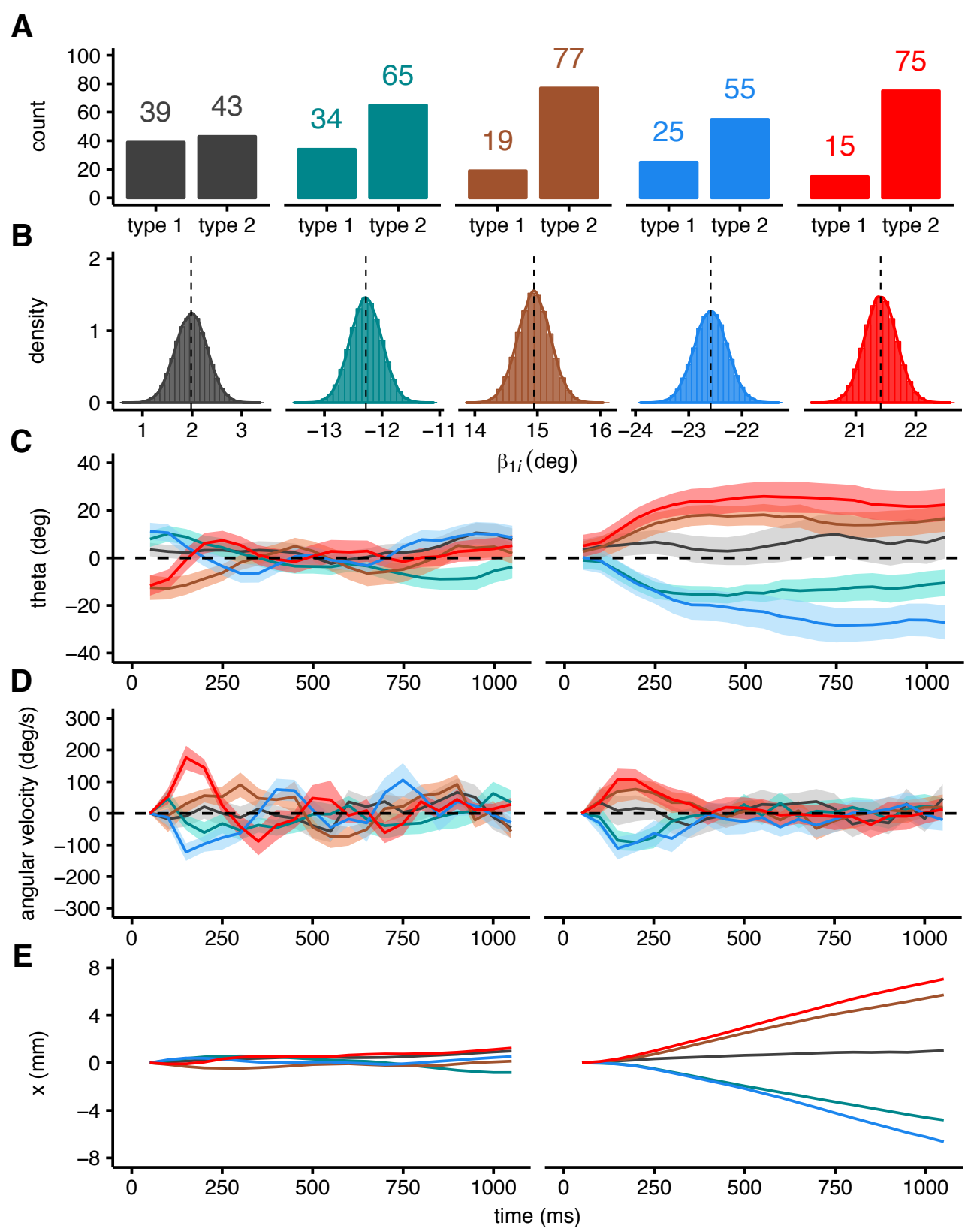




\section{Fig. 7}

Fig. 7. Trajectories split. (A) Count of the trials falling into the two types of trajectories by condition. Type 1 represents the trajectories in which the shift of the flies was at least $9 \mathrm{~mm}$ from an ideal straight line uniting the two Buridan stimuli when flies reached the middle of the path, while type 2 represents the trajectories for which the shift was less than $9 \mathrm{~mm}$. (B) Approximate density profile of the probability density function for the sampling distribution for the five conditions. Distributions show the likelihoods of the interaction parameters (between distance, condition and type). (C) Mean orientation of the flies during the first second by condition in the two types of trajectories. On the left are shown type 1 trajectories while on the right type 2 trajectories. The shaded region represents the s.e.m. (D) Mean of the angular velocity of the flies during the first 1000 milliseconds by condition in the two types of trajectories. Type 1 on the left, type 2 on the right. The shaded region represents the s.e.m. (E) Regression lines of the trajectories with the LOWESS (LOcally WEighted Scatterplot Smoothing) method during the first 1000 milliseconds by condition for the two types of trajectories. Type 1 on the left, type 2 on the right. In all images of the panel the color-coding is as previously described (see Fig. 3). 